\title{
Long non-coding RNA HOTAIR promotes cancer cell energy metabolism in pancreatic adenocarcinoma by upregulating hexokinase-2
}

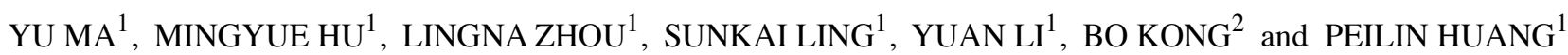 \\ ${ }^{1}$ Department of Oncology, School of Medicine, Southeast University, Nanjing, Jiangsu 210009, P.R. China; \\ ${ }^{2}$ Department of Surgery, School of Medicine, Technical University Munich, D-80333 Munich, Germany
}

Received March 3, 2018; Accepted February 15, 2019

DOI: $10.3892 / \mathrm{ol} .2019 .10551$

\begin{abstract}
Hox transcript antisense RNA (HOTAIR) is a long non-coding RNA (lncRNA) that serves a key role in the pathogenesis of various types of cancer, including pancreatic adenocarcinoma. However, the diagnostic and prognostic values of HOTAIR in pancreatic adenocarcinoma, as well as its involvement in cancer cell energy metabolism, remain unclear. In the present study, tumor tissues and adjacent healthy tissues were collected from patients with pancreatic adenocarcinoma, and blood samples were collected from patients and healthy controls. Expression levels of HOTAIR and hexokinase-2 (HK2) were detected by reverse transcription-quantitative polymerase chain reaction. All patients were followed up for 5 years, and the diagnostic and prognostic values of serum HOTAIR levels were investigated by receiver operating characteristic curve and survival analyses, respectively. Pancreatic adenocarcinoma cell lines overexpressing HOTAIR and HK2 were established, and the effects on cell proliferation, lactate production, glucose uptake and ATP production were detected by Cell Counting Kit- 8 , lactate, glucose uptake and ATP assays, respectively. The protein expression was detected by western blot analysis. The results revealed that HOTAIR and HK2 expression levels were increased in tumor tissues compared with adjacent healthy tissues. The serum levels of HOTAIR and HK2 were higher in patients with pancreatic cancer compared with healthy controls. The serum levels of these two factors may be used to accurately predict pancreatic adenocarcinoma and its prognosis. HOTAIR and HK2 overexpression led to the promotion of tumor cell proliferation. HOTAIR overexpression increased lactate production, glucose uptake and ATP production. Furthermore, it promoted HK2
\end{abstract}

Correspondence to: Dr Peilin Huang, Department of Oncology, School of Medicine, Southeast University, 87 Dingjiaqiao Road, Nanjing, Jiangsu 210009, P.R. China

E-mail: ed29e80hil2@163.com

Key words: pancreatic adenocarcinoma, energy metabolism, pancreatic cancer expression, however HK2 overexpression displayed no significant effects on HOTAIR expression levels. Therefore, it was concluded that the IncRNA HOTAIR may promote cancer cell energy metabolism in pancreatic adenocarcinoma by upregulating $\mathrm{HK} 2$.

\section{Introduction}

Pancreatic cancer is a malignancy that originates from cells in the pancreas, and can be divided into a number of subtypes, according to pathological features (1). These subtypes include intraductal papillary-mucinous neoplasm, acinar cell carcinoma, mucinous cystic neoplasm with an invasive adenocarcinoma and adenocarcinoma (1). Pancreatic adenocarcinoma, as the predominant type of pancreatic cancer, accounts for $>85 \%$ of all cases (1). Despite advances in the treatment and prevention of this disease, pancreatic cancer remains a major cause of cancer-associated mortalities and, in 2015 , it was reported to be directly responsible for $>200,000$ mortalities worldwide annually (2). The 5-year survival rate in 2012 in certain regions, including China was $<5 \%$, or $<1 \%$ in particular areas, for example the Southeast (3). In addition, the incidence rate of pancreatic adenocarcinoma has increased in the last 5 years worldwide (4). At present, surgical resection is the only radical treatment for pancreatic adenocarcinoma (4). However, it is difficult to detect early stage pancreatic adenocarcinoma and the majority of patients are diagnosed at advanced stages, making surgical resection an inappropriate treatment, leading to poor prognosis and high mortality rates (5). Male gender, older age, a poor diet, diabetes mellitus, obesity and smoking have been proven to be risk factors for pancreatic adenocarcinoma (6). However, the current understanding on the pathogenesis of pancreatic cancer is limited.

The development of pancreatic cancer is a complex process with numerous genetic factors involved. Additionally to messenger RNAs (mRNAs), which encode proteins, the human genome also transcribes a number of non-coding RNAs (ncRNAs) that can participate in physiological processes and pathological changes (7). As a subtype of ncRNAs, long ncRNAs (IncRNAs) have been demonstrated to be involved in the pathogenesis of several human diseases, including various types of malignacy (8). Since hox transcript antisense RNA 
(HOTAIR) was reported to function as an oncogene in breast cancer by modifying chromatin structure, this lncRNA has been demonstrated to participate in the occurrence, development and progression of various types of cancer, including pancreatic adenocarcinoma $(9,10)$. However, the diagnostic and prognostic values of HOTAIR for pancreatic adenocarcinoma, as well as its involvement in cancer cell energy metabolism requires further investigation.

\section{Materials and methods}

Patients. Between August 2009 and August 2012, a total of 78 patients with pancreatic adenocarcinoma at the Department of Oncology, Affiliated Hospital of School of Medicine, Southeast University (Nanjing, China) were enrolled to the present study. Pancreatic adenocarcinoma was confirmed in patients by pathological and imaging examinations. A total of 48 males and 30 females, with an mean age of $45.0 \pm 16.1$ years (range, 19-78 years), were included. Primary tumors were staged according to following standards: i) T1, tumor confined to the pancreas with a maximum diameter $<2 \mathrm{~cm}$; ii) $\mathrm{T} 2$, tumor confined to the pancreas with a diameter of $2-4 \mathrm{~cm}$; iii) $\mathrm{T} 3$, tumor confined to the pancreas with diameter $>4 \mathrm{~cm}$; and iv) T4, celiac axis or the superior mesenteric artery were involved. The cohort included 12 cases of stage 1,14 cases of stage 2, 25 cases of stage 3 and 27 cases of stage 4 (AJCC) (11). Among these patients, 51 received surgical resection, and tumor tissues, as well as adjacent healthy tissues, were collected during the procedure. All tissues were confirmed by pathological examinations. Additionally, 30 healthy volunteers (sex, 18 male and 12 female; age range, 20-79 years; mean, $45.3 \pm 15.2$ years) with similar age and gender distributions were enrolled, to serve as a control group. The Ethics Committee of the Affiliated Hospital of School of Medicine, Southeast University approved this study. All patients and volunteers provided signed informed consent.

Preparation of serum samples. Fasting blood $(20 \mathrm{ml})$ was collected from all 78 patients and 30 healthy controls on the morning following their admission. The blood samples were incubated at room temperature for $1.5 \mathrm{~h}$, followed by centrifugation at $1,400 \mathrm{x} \mathrm{g}$ at room temperature for $15 \mathrm{~min}$ to separate the serum, which was then stored at $-80^{\circ} \mathrm{C}$ prior to use.

Cell lines and cell culture. Human pancreatic adenocarcinoma cell lines BxPC-3 and Capan-2 were purchased from the American Type Culture Collection (ATCC; Manassas, VA, USA). All cell lines were cultured according to the supplier's protocols. The BxPC-3 cells were cultured in ATCC-formulated RPMI-1640 medium containing 10\% fetal bovine serum (FBS) (both ATCC). The Capan-2 cells were cultured in ATCC-formulated McCoy's 5a medium (ATCC) containing $10 \% \mathrm{FBS}$. All incubations were performed at $37^{\circ} \mathrm{C}$. Cells were harvested during logarithmic growth phase for subsequent experiments.

Establishment of HOTAIR- and hexokinase-2 (HK2)overexpressing cell lines. HOTAIR (sequence ID, NR_047517.1) and HK2 (GeneBank ID, M23115.1) expression vectors (pcDNA3.1) were established by inserting an
EcoRI fragment containing full-length HOTAIR or HK2 cDNA into the pcDNA3.1 vector (Clontech Laboratories, Inc., Mountainview, CA, USA). BxPC-3 and Capan-2 cells were cultured overnight to ensure they reached $80-90 \%$ confluence prior to transfection. The experiment was performed using Lipofectamine ${ }^{\circledR} 2000$ transfection reagent (Invitrogen; Thermo Fisher Scientific, Inc., Waltham, MA, USA) to transfect $10 \mathrm{nM}$ vector into $10^{6}$ cells, according to the manufacturer's protocol. Empty vector without HOTAIR or HK2 cDNA was used as negative control. The interval between transfection and subsequent experiments was $24 \mathrm{~h}$.

Cell proliferation assay. For the cell proliferation assays, a Cell Counting Kit-8 (CCK-8; Dojindo Molecular Technologies, Inc., Kumamoto, Japan) was used. BxPC-3 and Capan-2 cells were plated into a 96 -well plate (100 $\mu \mathrm{l}$ cell suspension containing $4 \times 10^{4}$ cells), cultured at $37^{\circ} \mathrm{C}$, and CCK-8 solution $(10 \mu \mathrm{l})$ was added into each well at $24,48,72$ and $96 \mathrm{~h}$ post-cell seeding. Following the addition of CCK-8 solution, cells were cultured at $37^{\circ} \mathrm{C}$ for an additional $4 \mathrm{~h}$, and a Fisherbrand ${ }^{\mathrm{TM}}$ accuSkan $^{\mathrm{TM}}$ GO UV/Vis microplate spectrophotometer (Thermo Fisher Scientific, Inc.) was used to measure optical density (OD) values at a wavelength of $450 \mathrm{~nm}$.

Lactate production, glucose uptake and ATP production assays. The ATP assay kit, Celltiter-Glo Luminescent Cell Viability assay (Promega Corporation, Madison, WI, USA) was used to measure ATP levels, according to the manufacturer's protocols. For the glucose uptake assays, BxPC-3 and Capan-2 cells were cultured in 6-well plates for $24 \mathrm{~h}$ at a density of $\sim 5 \times 10^{5}$ cells per well. The cell supernatant was collected and OD values at $570 \mathrm{~nm}$ were measured with a microplate reader, using fresh culture medium as a control. Glucose uptake was calculated by subtracting the concentration of glucose in the tested samples from the initial concentration of glucose in the fresh culture medium. Lactate production was determined according to a standard curve. Lactate production and glucose uptake were normalized to the cellular protein levels.

Reverse transcription-quantitative polymerase chain reaction $(R T-q P C R)$. TRIzol ${ }^{\circledR}$ reagent (Invitrogen; Thermo Fisher Scientific Inc.) was used to extract total RNA from tumor tissues, adjacent healthy tissues, serum and in vitro cultured cells. Tumor tissues and adjacent healthy tissues were ground in liquid nitrogen prior to the addition of TRIzol. RNA quality was tested using gel electrophoresis, and the RNA samples were subjected to RT for the synthesis of cDNA using AMV Reverse Transcriptase XL kit (Takara Bio, Inc., Otsu, Japan). The temperature conditions were $25^{\circ} \mathrm{C}$ for $5 \mathrm{~min}, 55^{\circ} \mathrm{C}$ for $15 \mathrm{~min}$ and $75^{\circ} \mathrm{C}$ for $10 \mathrm{~min}$. SYBR ${ }^{\circledR}$ Green Real-Time PCR master mix (Thermo Fisher Scientific, Inc.) was used for qPCR reactions with the cDNA, according to the manufacturer's protocols. The following primers were used: HOTAIR forward, 5'-AACGATGTGTGTGTGCCTTGAT-3'; and reverse, 5'-TGGTCCGACAGGGTGAATT-3'; HK2 forward, 5'-GGGCATCTTGAAACAAG-3'; and reverse, 5'-GGTCTC AAGCCCTAAG-3'; and $\beta$-actin forward, 5'-GACCTCTAT GCCAACACAGT-3'; and reverse, 5'-AGTACTTGCGCT CAGGAGGA-3'. The thermocycling conditions were: $95^{\circ} \mathrm{C}$ for $50 \mathrm{~s}$, followed by 40 cycles of $95^{\circ} \mathrm{C}$ for $12 \mathrm{~s}$ and $60^{\circ} \mathrm{C}$ for 
$30 \mathrm{~s}$. The data were processed using the $2^{-\Delta \Delta \mathrm{Cq}}$ method (12). The expression levels of HOTAIR and HK2 were normalized to the endogenous $\beta$-actin control levels.

Western blot analysis. Radioimmunoprecipitation assay buffer (Thermo Fisher Scientific, Inc.) was used to extract total protein from BxPC-3 and Capan-2 cells, and protein quantity was determined by bicinchoninic acid assay. Gel electrophoresis was performed using $10 \%$ SDS-PAGE with $25 \mu \mathrm{g}$ per lane and the proteins were transferred onto polyvinylidene difluoride membranes. The membranes were blocked with 5\% skimmed milk for $2 \mathrm{~h}$ at room temperature, followed by incubation with primary antibodies including rabbit anti-HK2 (dilution, 1:2,000; cat. no. ab37593) and anti-GAPDH primary antibody (dilution, 1:1,000; cat. no. ab8245) (both Abcam, Cambridge, MA, USA) overnight at $4^{\circ} \mathrm{C}$. The following day, membranes were washed in triplicate with PBS for $15 \mathrm{~min}$, and incubated with horseradish peroxidase-conjugated anti-rabbit IgG secondary antibody (dilution, 1:1,000; cat. no. MBS435036; MyBioSource, Inc., San Diego, CA, USA) at room temperature for $2 \mathrm{~h}$, followed by signal detection using an enhanced chemiluminescence kit (Sigma-Aldrich; Merck KGaA, Darmstadt, Germany). Signals were scanned using MYECL ${ }^{\mathrm{TM}}$ Imager (Thermo Fisher Scientific, Inc.), and ImageJ v.1.48 software (National Institutes of Health, Bethesda, MD, USA) was used to normalize the relative expression level of HK2 to the endogenous GAPDH control levels.

Statistical analysis. All data were processed using SPSS version 19.0 (IBM Corp., Armonk, NY USA). Count data were processed using a $\chi^{2}$ test. Measurement data were expressed as mean \pm standard deviation, and comparisons between two groups were performed using Student's paired t-test. Comparisons among multiple groups were performed using analysis of variance (one-way) and a least significant difference post hoc test. Receiver operating characteristic (ROC) curve analysis was performed to evaluate the diagnostic value of serum HOTAIR and HK2 levels in pancreatic adenocarcinoma. Patients were divided into high- and low-expression groups according to the median serum levels of HOTAIR and HK2. Survival curves were plotted using the Kaplan-Meier method and were compared using a log rank test. $\mathrm{P}<0.05$ was considered to indicate a statistically significant difference.

\section{Results}

Expression of HOTAIR and HK2 in tumor and adjacent healthy tissues of patients with pancreatic adenocarcinoma. The expression levels of HOTAIR and HK2 in tumor and adjacent healthy tissues of 51 patients with pancreatic adenocarcinoma, who had undergone surgical resection, were detected by RT-qPCR. As indicated in Fig. 1A, the expression level of HOTAIR was significantly upregulated in tumor tissues compared with adjacent healthy tissues $(\mathrm{P}<0.05)$ in 46/51 patients. Decreased HOTAIR expression levels in tumor tissues compared with those in the adjacent healthy tissues $(\mathrm{P}<0.05)$ were observed in only $2 / 51$ patients, and no significant difference was indicated in 4/51 patients. Similarly, the mRNA levels of HK2 were significantly upregulated in tumor tissues compared with adjacent healthy tissues $(\mathrm{P}<0.05)$ in 41/51 patients. Decreased HK2 levels of in tumor tissues compared with the adjacent healthy tissues $(\mathrm{P}<0.05)$ were only observed in $4 / 51$ cases, and no significant difference was observed in 6/51 cases (Fig. 1B). These results suggest that the upregulation of HOTAIR and HK2 is involved in the pathogenesis of pancreatic adenocarcinoma.

Serum levels of HOTAIR and HK2 in healthy controls and patients with different stages of pancreatic adenocarcinoma. As presented in Fig. 2A, the expression levels of HOTAIR were significantly lower in the serum of healthy controls compared with patients with different stages of pancreatic adenocarcinoma $(\mathrm{P}<0.05)$. In addition, the expression levels of HOTAIR were further increased at more advanced stages of primary tumor. Similarly, serum levels of HK2 were significantly higher in patients with different stages of pancreatic adenocarcinoma compared with healthy controls (Fig. 2B; $\mathrm{P}<0.05)$. In addition, expression levels of HK2 were increased with the later stages of primary tumors.

Diagnostic and prognostic value of HOTAIR and HK2 in pancreatic adenocarcinoma. ROC curve analysis was performed in order to evaluate the diagnostic value of serum HOTAIR and HK2 levels in pancreatic adenocarcinoma. The area under the curve for serum HOTAIR levels was 0.9329 [95\% confidence interval (CI), 0.8888-0.9770; $\mathrm{P}<0.0001$; Fig. 3A], and for serum HK2 it was 0.8893 (95\% CI, 0.8295-0.9492; P<0.0001; Fig. 3B). These data suggest that serum HOTAIR and HK2 levels may serve as potential biomarkers in the diagnosis of pancreatic adenocarcinoma. Patients were divided into high- and low-expression groups, according to the median serum levels of HOTAIR and HK2. Survival curves were plotted using the Kaplan-Meier method and were compared using a log rank test. As presented in Fig. $3 \mathrm{C}$ and $\mathrm{D}$, the overall survival rate of patients with high expression levels of HOTAIR or HK2 was significantly lower compared with that of patients with low expression levels $(\mathrm{P}=0.011$ and $\mathrm{P}=0.014$, respectively). These data suggest that serum HOTAIR and HK2 may serve as promising diagnostic and prognostic biomarkers for pancreatic adenocarcinoma.

HOTAIR and HK2 overexpression promotes the proliferation of pancreatic adenocarcinoma. HOTAIR and HK2 overexpression in pancreatic adenocarcinoma BxPC-3 and Capan-2 cells following transfection was confirmed by RT-qPCR (Fig. 4A; $\mathrm{P}<0.05$ ). As indicated in Fig. 4B, compared with the equivalent control cells, HOTAIR and HK2 overexpression appeared to promote the proliferation of the two pancreatic adenocarcinoma cell types $(\mathrm{P}<0.05)$, and the enhancing effect of HK2 overexpression on the proliferation rate was stronger than that of HOTAIR overexpression, but the differences were not statistically significant. These findings indicate that HOTAIR and HK2 overexpression can promote the proliferation of pancreatic adenocarcinoma cells.

HOTAIR is an upstream regulator of HK2 in pancreatic adenocarcinoma. A recent study reported that HOTAIR can interact with HK2 to achieve its biological functions (12). In the present study, HOTAIR overexpression was revealed to lead to a significant increase the level of HK2 protein in 

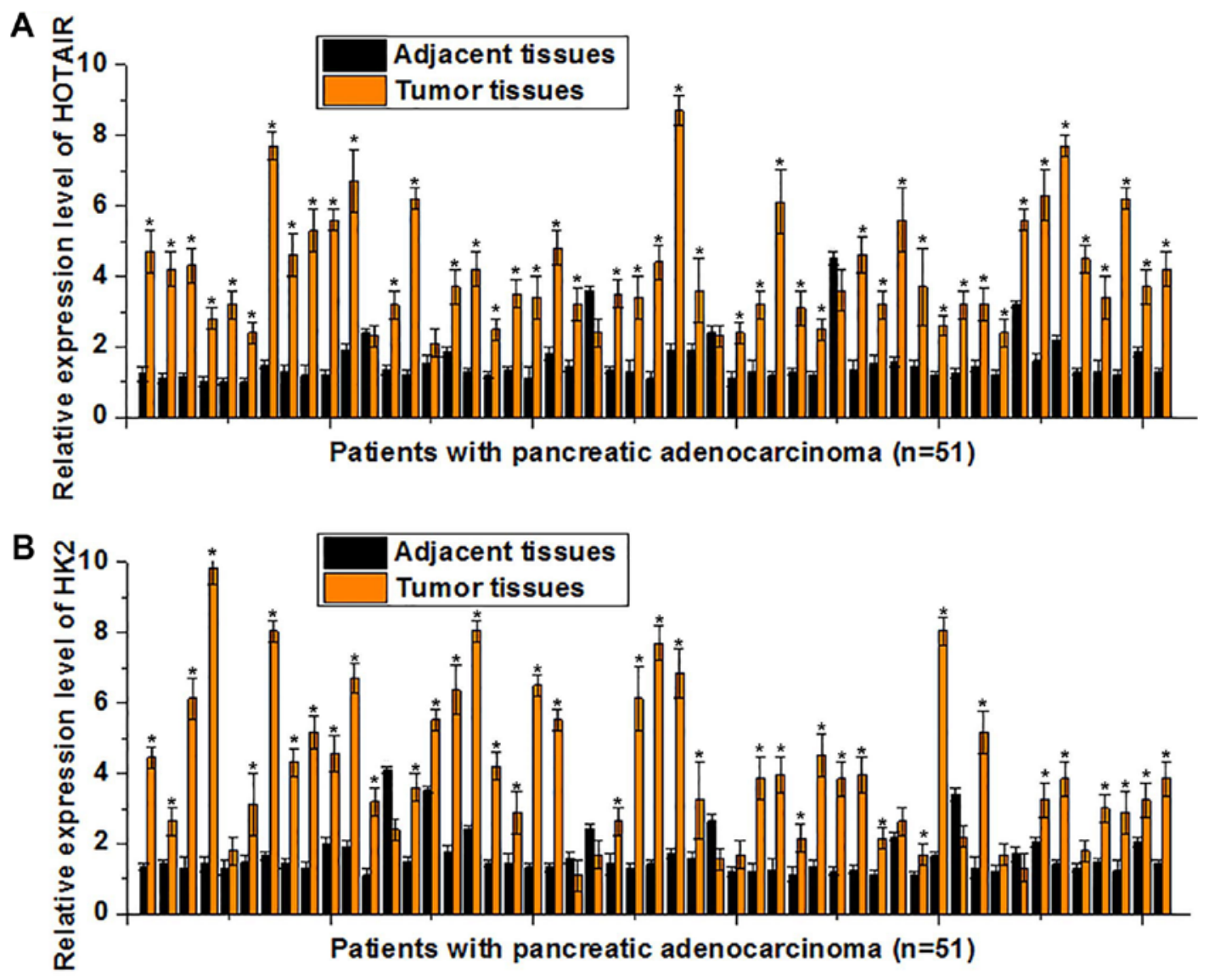

Figure 1. Expression of HOTAIR and HK2 in tumor and adjacent healthy tissues of patients with pancreatic adenocarcinoma. Relative expression levels of (A) HOTAIR and (B) HK2 in tumor tissues and adjacent healthy tissues of patients with pancreatic adenocarcinoma (n=51). qPCR was repeated 3 times and data were presented as mean \pm standard deviation. ${ }^{*} \mathrm{P}<0.05$ HK2, hexokinase-2; HOTAIR, hox transcript antisense RNA.

A

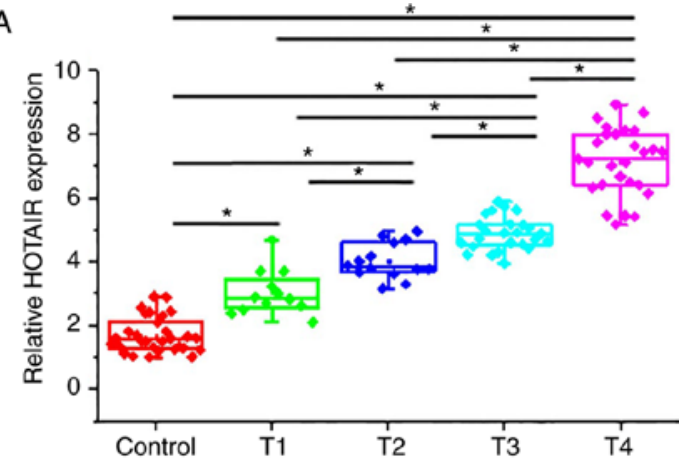

B

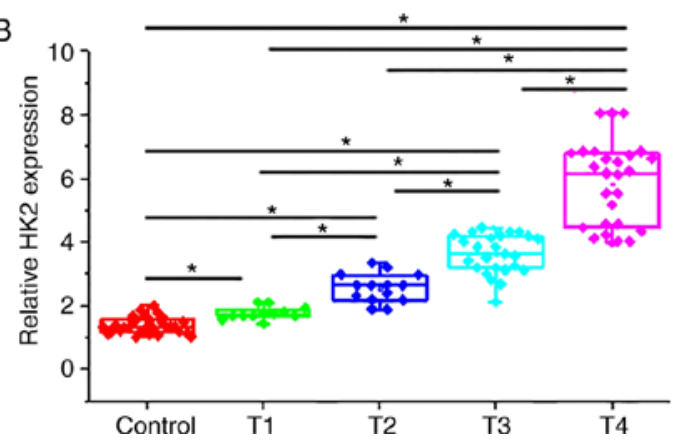

Figure 2. Serum levels of HOTAIR and HK2 in healthy controls and patients with pancreatic adenocarcinoma. Relative expression levels of (A) HOTAIR and (B) HK2 in the serum of healthy controls and patients with different stages of pancreatic adenocarcinoma. ${ }^{*} \mathrm{P}<0.05$. HK2, hexokinase-2; HOTAIR, hox transcript antisense RNA; T1-4, tumor stages, as defined in the Materials and methods section. the two tested pancreatic adenocarcinoma cell lines $(\mathrm{P}<0.05$; Fig. 5A and B). By contrast, HK2 overexpression led to no observed significant effects on the expression of HOTAIR ( $\mathrm{P}>0.05$; Fig. 5C). These results suggest that HOTAIR is an upstream regulator of $\mathrm{HK} 2$ in these cells.

HOTAIR overexpression increases lactate production, glucose uptake and ATP production in pancreatic adenocarcinoma cells. As indicated in Fig. 6A, compared with the equivalent control cells, glucose uptake was significantly increased in the cells of both pancreatic adenocarcinoma cell lines following HOTAIR overexpression $(\mathrm{P}<0.05)$. Similarly, lactate production (Fig. 6B) and ATP production (Fig. 6C) were significantly increased following HOTAIR overexpression. This suggests that lncRNA HOTAIR may promote cancer cell energy metabolism in pancreatic adenocarcinoma.

\section{Discussion}

Pancreatic cancer, predominantly referred to as pancreatic adenocarcinoma, is thought to be the most lethal type of cancer, with a 1.5-2-fold higher incidence rate in males compared with females (13). In the present study, a total of 78 patients with pancreatic adenocarcinoma with a male to female ratio of 1.6:1 were enrolled. The occurrence, development and progression of pancreatic adenocarcinoma involve various genetic factors, including lncRNAs (14). In a recent study, Jiang et al (15) 

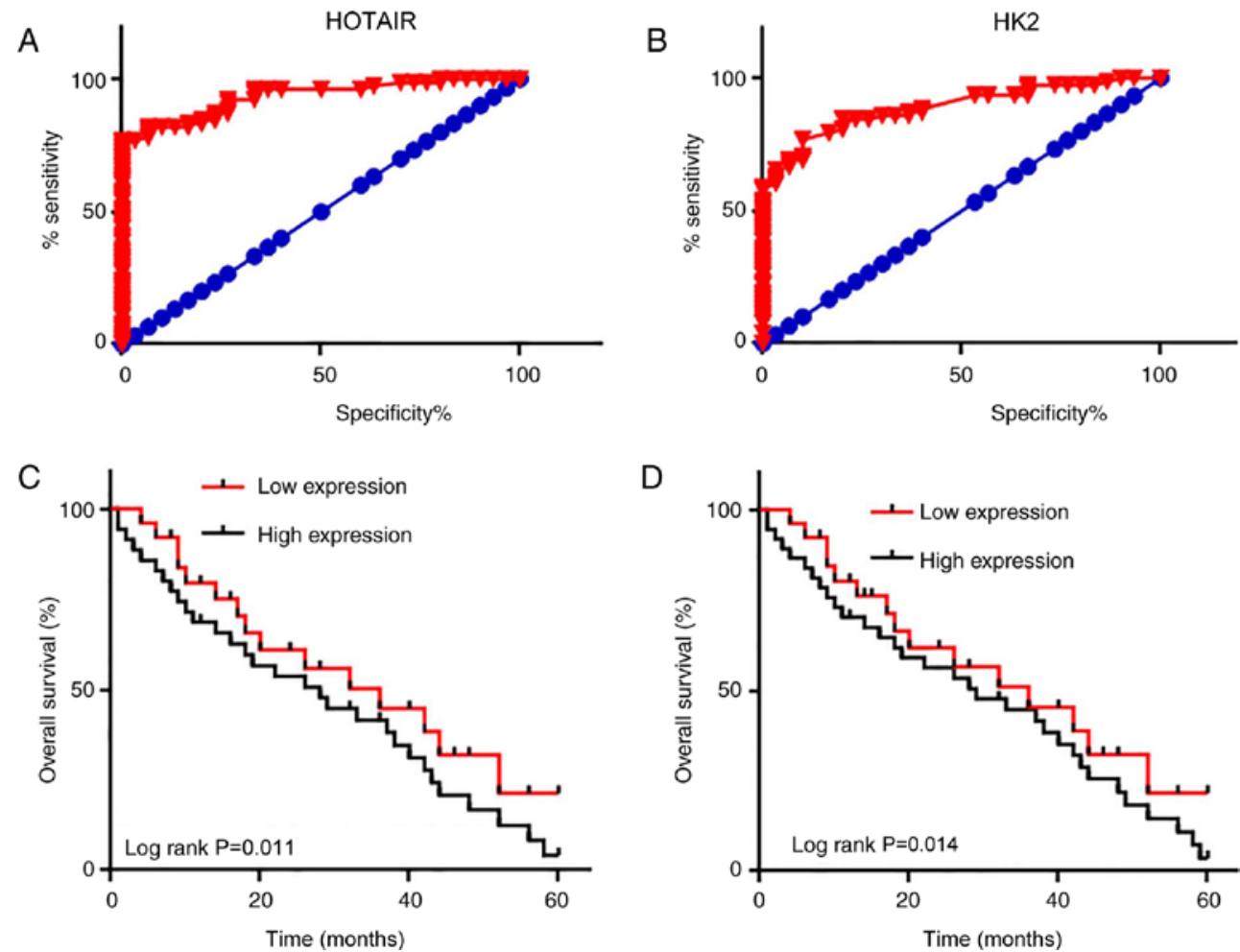

Figure 3. Diagnostic and prognostic value of HOTAIR and HK2 for pancreatic adenocarcinoma. Diagnostic value of serum (A) HOTAIR and (B) HK2 levels analyzed by receiver operating characteristic curve analysis. The blue line represents identity \% and the red line represents sensitivity \%. (C) Comparison of overall survival rates of patients with high vs. low serum levels of (C) HOTAIR and (D) HK2. HK2, HOTAIR and hexokinase-2; HOTAIR, hox transcript antisense RNA.

A
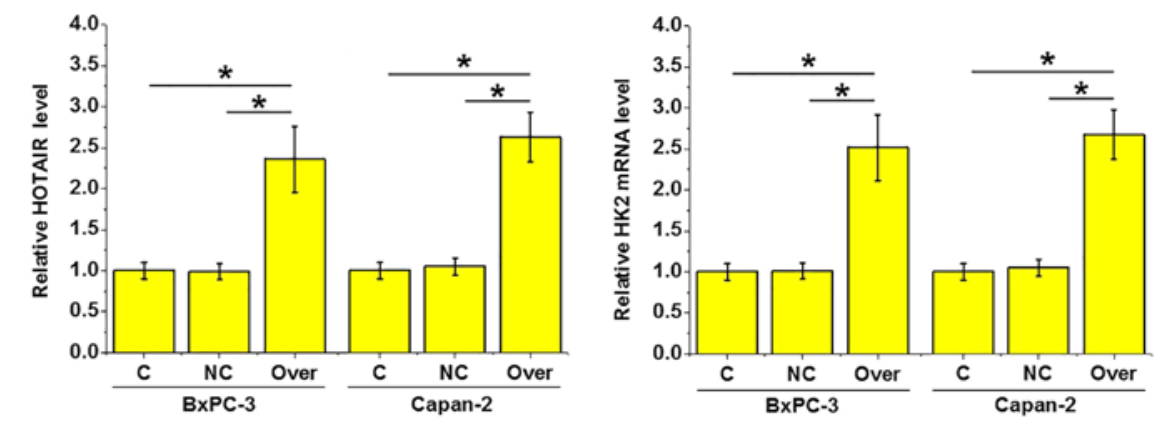

B
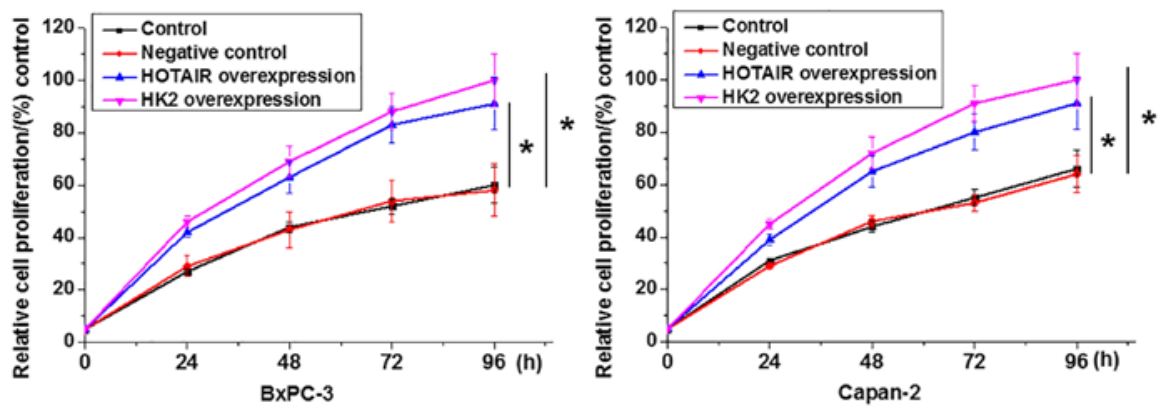

Figure 4. HOTAIR and HK2 overexpression promote pancreatic adenocarcinoma cell proliferation. (A) Confirmation of overexpression of HOTAIR and HK2 in BxPC-3 and Capan-2 cells by measuring their relative expression levels. ${ }^{*} \mathrm{P}<0.05$. (B) Normalized proliferation rate of BxPC-3 and Capan-2 cells with $\mathrm{HK} 2$ or HOTAIR overexpression * $\mathrm{P}<0.05$ at $96 \mathrm{~h}$. HK2, hexokinase-2; HOTAIR, hox transcript antisense RNA; C, healthy control; NC, empty vector control; Over, overexpression of HOTAIR of HK2.

reported that lncRNA HOTAIR was upregulated in pancreatic ductal adenocarcinoma tissue samples compared with adjacent healthy tissues. However, in another study, epigenetic silencing of HOTAIR was proved to be at least partially responsible for 
A
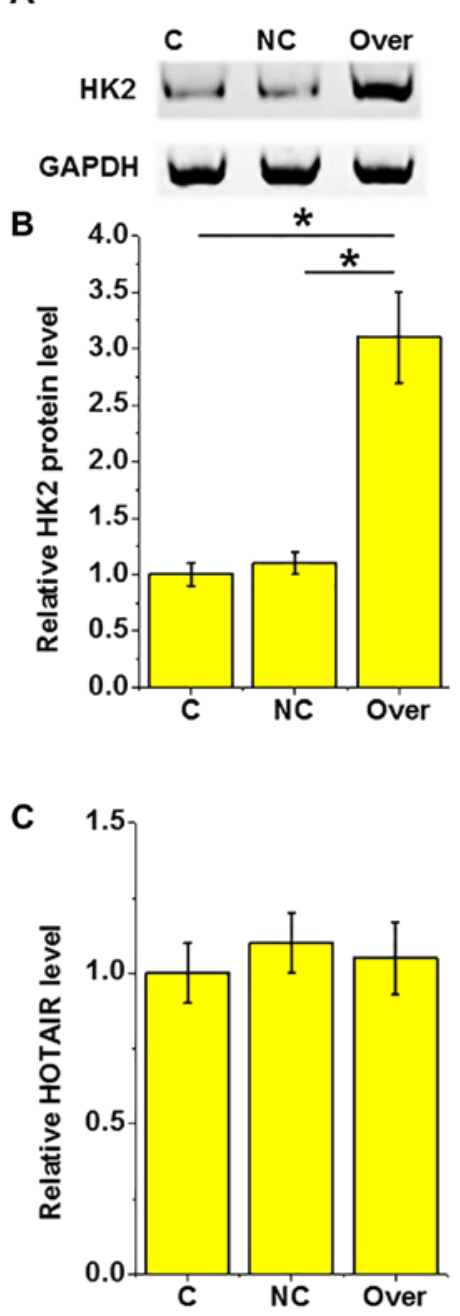

Capan-2
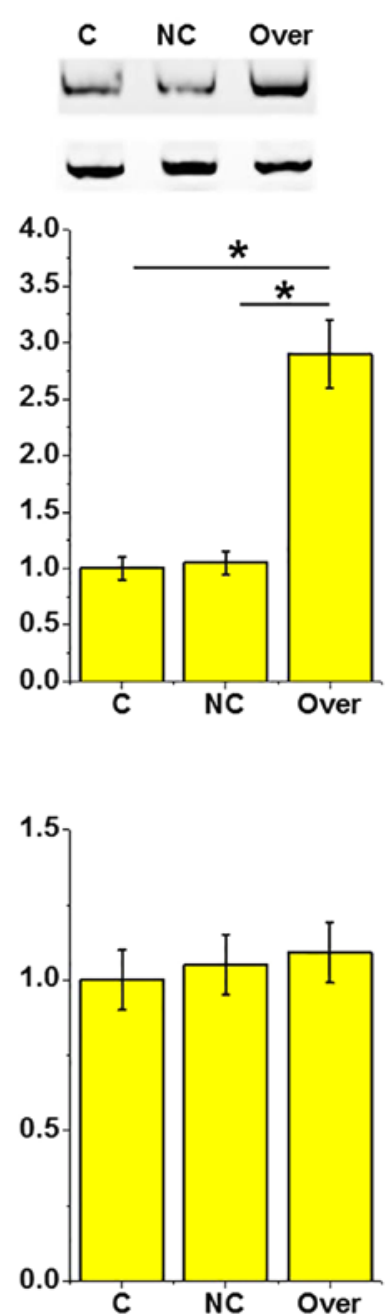

Figure 5. The interaction between HOTAIR and HK2. (A) Representative western blot images of HK2 detection from protein extracts of BxPC-3 and Capan-2 cell with overexpression of HOTAIR. (B) Normalized protein expression levels of HK2 in BxPC-3 and Capan-2 cells with overexpression of HOTAIR. (C) Normalized expression levels of HOTAIR expression in BxPC-3 and Capan-2 cells with overexpression of HK2 measured using RT-qPCR. " $\mathrm{P}<0.05$. HK2, hexokinase-2; HOTAIR, hox transcript antisense RNA; C, healthy control; NC, empty vector control; Over, overexpression of HOTAIR or HK2.

the metastasis of pancreatic cancer (16). The present results revealed that the expression of HOTAIR was significantly upregulated in tumor tissues compared with adjacent healthy tissues in 46 out of 51 patients, whereas downregulation was observed in only 2 cases. Altered metabolic processes are usually observed in cancer cells, and the characteristic changes may include enhanced rates of glutaminolysis and fatty acid synthesis, as well as increased uptake of glucose, which can support the proliferation and survival of tumor cells (17). HK2 is the major isozyme in aerobic glycolysis that is upregulated in various types of cancer, including liver and gastric cancer (18). In the present study, HK2 was also revealed to be upregulated in tumor tissues compared with adjacent healthy tissues in the majority of patients with pancreatic adenocarcinoma. In addition, the expression levels of HOTAIR and HK2 were increased as the primary tumor stage advanced. These findings suggest that the upregulation of HOTAIR and HK2

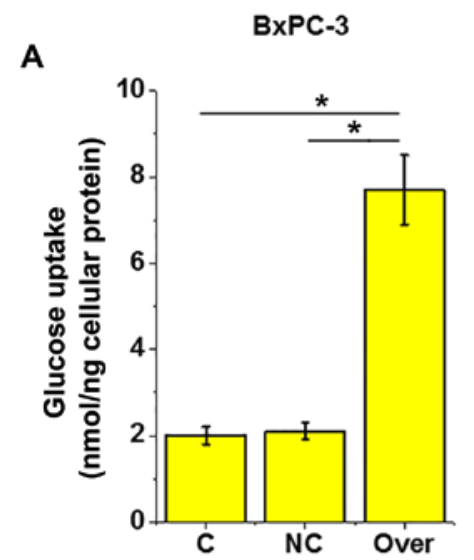

Capan-2

B
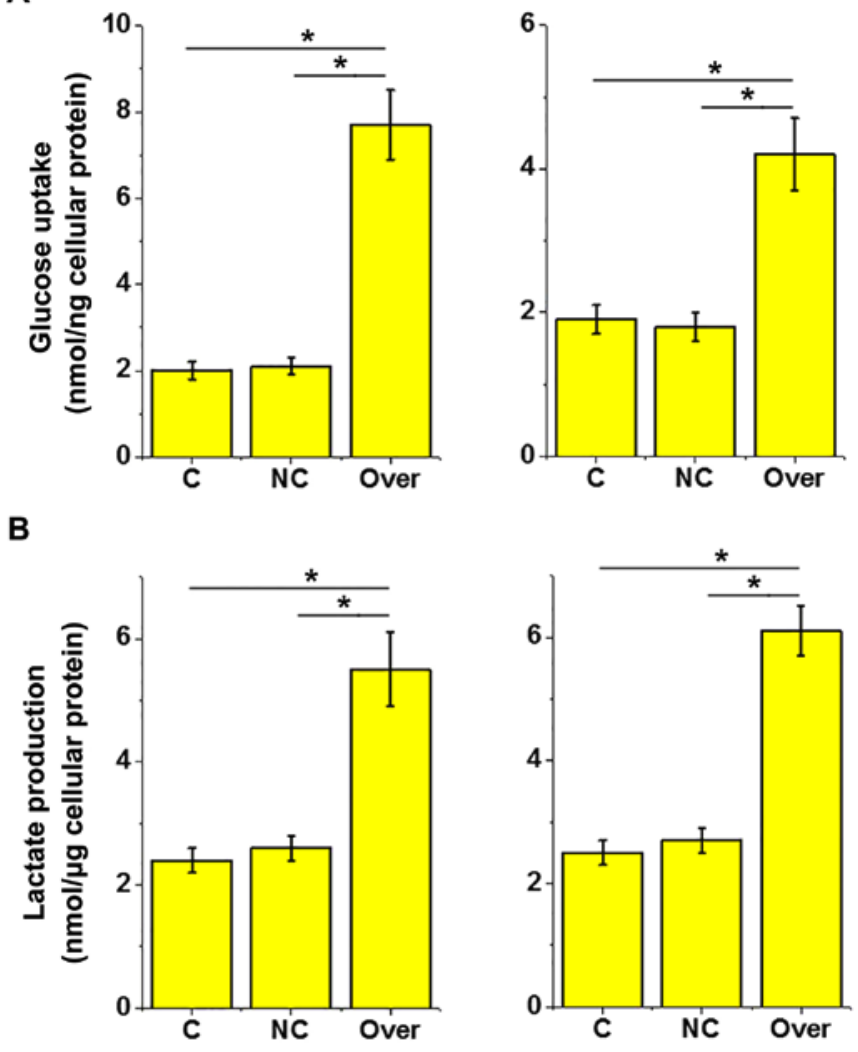

C
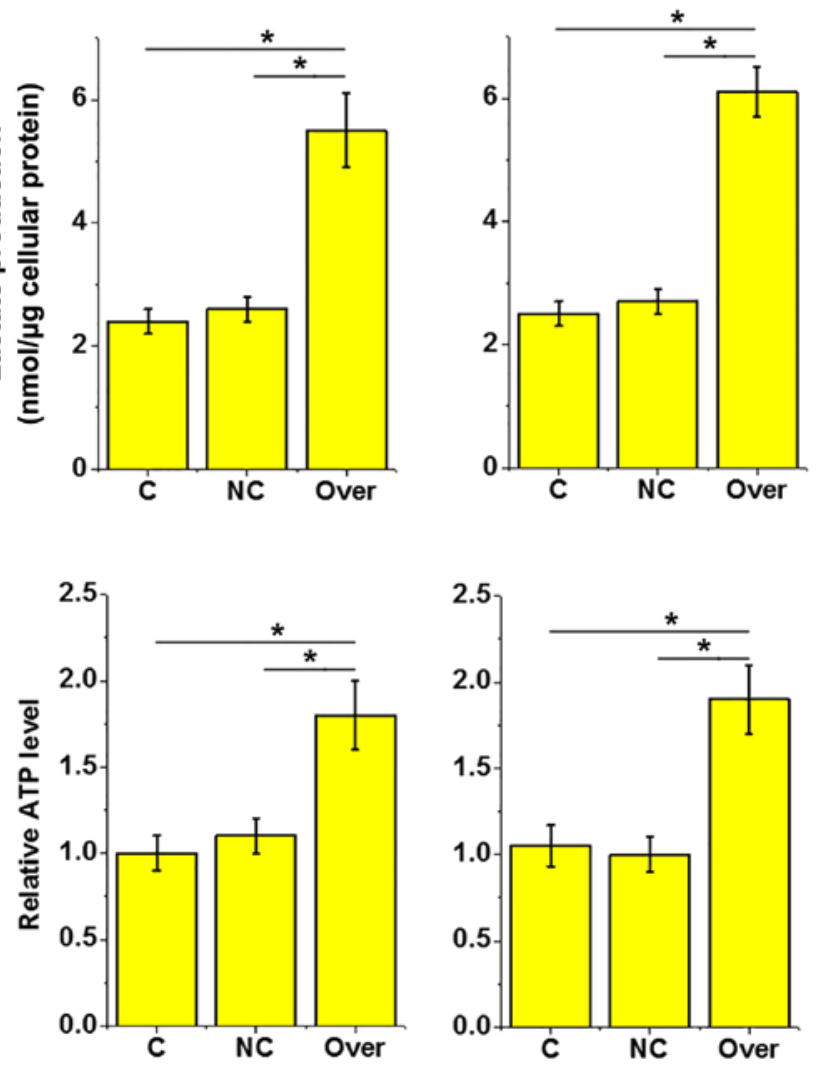

Figure 6. HOTAIR overexpression increases lactate production, glucose uptake and ATP production in pancreatic adenocarcinoma cells. Measurement of (A) glucose uptake, (B) lactate production and (C) ATP production in BxPC-3 and Capan-2 cells with overexpression of HOTAIR. ${ }^{\text {*P }}<0.05$. HOTAIR, hox transcript antisense RNA; C, healthy control; NC, empty vector of HOTAIR; Over, overexpression of HOTAIR.

is involved in the development and progression of pancreatic adenocarcinoma.

At the present time, surgical resection remains the only radical treatment for pancreatic adenocarcinoma (13). However, the majority of pancreatic adenocarcinoma cases are diagnosed at advanced stages due to lack of obvious symptoms earlier on, making surgical resection no longer an option for these patients (5). Therefore, early diagnosis and treatment remain key for the management of this disease. In the present study, ROC curve analyses indicated that HOTAIR and HK2 could be employed to accurately detect pancreatic adenocarcinoma. In addition, the overall survival 
rate of patients with high expression levels of HOTAIR or HK2 was significantly lower than that of patients with low expression. These data suggest that HOTAIR and HK2 may serve as diagnostic and prognostic biomarkers of pancreatic adenocarcinoma. However, HOTAIR and HK2 have also been reported to be of diagnostic and prognostic value in numerous other types of malignancy, inevitably affecting their specificity $(15,16,18)$. Therefore, the combination of multiple biomarkers may improve the diagnosis and prognosis of pancreatic adenocarcinoma.

HOTAIR and HK2 have been reported to serve a critical role in the proliferation of cancer cells and development of the disease $(19,20)$. In the present study, compared with their equivalent controls, HOTAIR and HK2 overexpression significantly promoted the proliferation of the two tested pancreatic adenocarcinoma cell lines, BxPC-3 and Capan-2. Despite the fact that the function of HOTAIR has been studied in various types of cancer, including pancreatic cancer $(15,16)$, its involvement in cancer cell energy metabolism, which is critical for cell growth and proliferation, remains unclear. Until now, a number of lncRNAs have been demonstrated to participate in glucose metabolism in cancer (20), and Cao et al (21) proposed an electron-transfer equilibrium with a small reorganization energy in aerobic glycolysis by using synthetic model systems. In the present study, HOTAIR overexpression significantly increased lactate production, glucose uptake and ATP production in pancreatic adenocarcinoma cells, indicating that it can promote energy metabolism in these cells. HK2 serves a key role in aerobic glycolysis by regulating lactate production, glucose uptake and ATP production (17), and aerobic glycolysis is regulated by HOTAIR in the pathogenesis of certain human diseases, including esophageal squamous cell carcinoma (12). The present findings demonstrated that HOTAIR overexpression led to a significant increase in the expression of HK2 in BxPC-3 and Capan-2 cells, whereas HK2 overexpression revealed no significant effects on HOTAIR levels. These results suggest that HOTAIR is an upstream regulator of HK2 in pancreatic adenocarcinoma.

In conclusion, HOTAIR and HK2 expression levels were increased in tumor tissues compared with adjacent healthy tissues in the majority of patients with pancreatic adenocarcinoma. Serum levels of these two factors were higher in patients with this cancer than in healthy controls, and increased with tumor progression. Serum HOTAIR and HK2 may be used to accurately detect pancreatic adenocarcinoma and predict its prognosis. Overexpression of these two molecules promoted tumor cell proliferation, and HOTAIR upregulation led to increased lactate production, glucose uptake and ATP production in pancreatic adenocarcinoma cells. HOTAIR overexpression promoted HK2 expression, however, HK2 upregulation exhibited no significant effects on HOTAIR. Therefore, it can be concluded that lncRNA HOTAIR may promote cancer cell energy metabolism in pancreatic adenocarcinoma by serving as a positive upstream regulator of $\mathrm{HK} 2$.

\section{Acknowledgements}

Not applicable.

\section{Funding}

The present study was funded by Postgraduate Research and Practice Innovation Program of Jiangsu Province (grant no. KYCX17_0183).

\section{Availability of data and materials}

The datasets generated and/or analyzed during the study are available from the corresponding author on reasonable request.

\section{Authors' contributions}

YM and PH designed experiments. YM, MH, LZ performed experiments. SL, YL and BK analyzed data and assisted the experiments. PH drafted the manuscript. All authors approved this manuscript.

\section{Ethics approval and consent to participate}

This study was approved by the Ethics Committee of Affiliated Hospital of School of Medicine, Southeast University.

\section{Patient consent for publication}

Patients provided consent for publication of their information.

\section{Competing interests}

The authors declare that they have no competing interests.

\section{References}

1. Stewart BW and Wild CP: World cancer report 2014. Health, 2017.

2. Chen W, Zheng R, Baade PD, Zhang S, Zeng H, Bray F, Jemal A. $\mathrm{Yu}$ XQ and He J: Cancer statistics in China, 2015. CA Cancer J Clin 66: 115-132, 2016.

3. Chen W, Zheng R, Zuo T, Zeng H, Zhang S and He J: National cancer incidence and mortality in China, 2012. Chin J Cancer Res 28: 1-11, 2016.

4. Ryan DP, Hong TS and Bardeesy N: Pancreatic adenocarcinoma. N Engl J Med 371: 1039-1049, 2014.

5. Sharma C, Eltawil KM, Renfrew PD, Walsh MJ and Molinari M Advances in diagnosis, treatment and palliation of pancreatic carcinoma: 1990-2010. World J Gastroenterol 17: 867-897, 2011.

6. Vincent A, Herman J, Schulick R, Hruban RH and Goggins M: Pancreatic cancer. Lancet 378: 607-620, 2011.

7. Mattick JS and Makunin IV: Non-coding RNA. Hum Mol Genet 15 (Spec No 1): R17-R29, 2006.

8. Cui Z, Ren S, Lu J, Wang F, Xu W, Sun Y, Wei M, Chen J, Gao X, $\mathrm{Xu} \mathrm{C}$, et al: The prostate cancer-up-regulated long noncoding RNA PlncRNA-1 modulates apoptosis and proliferation through reciprocal regulation of androgen receptor. Urol Oncol 31: 1117-1123, 2013.

9. Gupta RA, Shah N, Wang KC, Kim J, Horlings HM, Wong DJ, Tsai MC, Hung T, Argani P, Rinn JL, et al: Long non-coding RNA HOTAIR reprograms chromatin state to promote cancer metastasis. Nature 464: 1071-1076, 2010.

10. Cai H, Yao J, An Y, Chen X, Chen W, Wu D, Luo B, Yang Y, Jiang Y, Sun D and He X: LncRNA HOTAIR acts as competing endogenous RNA to control the expression of Notch3 via sponging miR-613 in pancreatic cancer. Oncotarget 8: 32905-32917, 2017.

11. Kamarajah SK, Burns WR, Frankel TL, Cho CS and Nathan H: Validation of the American Joint Commission on Cancer (AJCC) 8th edition staging system for patients with pancreatic adenocarcinoma: A Surveillance, Epidemiology and End Results (SEER) Analysis. Ann Surg Oncol 24: 2023-2030, 2017. 
12. Livak KJ and Schmittgen TD: Analysis of relative gene expression data using real-time quantitative PCR and the 2(-Delta Delta C(T)) method. Methods 25: 402-408, 2001

13. Siegel R, Ma J, Zou Z and Jemal A: Cancer statistics, 2014. CA Cancer J Clin 64: 9-29, 2014.

14. Pang EJ, Yang R, Fu XB and Liu YF: Overexpression of long non-coding RNA MALAT1 is correlated with clinical progression and unfavorable prognosis in pancreatic cancer. Tumour Biol 36: 2403-2407, 2015

15. Jiang Y, Li Z, Zheng S, Chen H, Zhao X, Gao W, Bi Z, You K, Wang Y, Li W, et al: The long non-coding RNA HOTAIR affects the radiosensitivity of pancreatic ductal adenocarcinoma by regulating the expression of Wnt inhibitory factor 1 . Tumour Biol 37: 3957-3967, 2016.

16. Nie H, Zhang Y, Xing R, Li M and Mou Y: Epigenetic silence of HOTAIR contributes to the metastasis of pancreatic cancer via targeting miR-138. Oncol Res: Dec 21, 2017 (Epub ahead of print).

17. Fadaka A, Ajiboye B, Ojo O, Adewale O and Olayide I: Biology of glucose metabolization in cancer cells. J Oncol Sci 3: 45-51, 2017.

18. Mathupala SP, Ko YH and Pedersen PL: Hexokinase-2 bound to mitochondria: Cancer's stygian link to the 'Warburg Effect' and a pivotal target for effective therapy. Semin Cancer Biol 19: 17-24, 2009.
19. Katagiri M, Karasawa H, Takagi K, Nakayama S, Yabuuchi S, Fujishima F, Naitoh T, Watanabe M, Suzuki T, Unno M and Sasano H: Hexokinase 2 in colorectal cancer: A potent prognostic factor associated with glycolysis, proliferation and migration. Histol Histopathol 32: 351-360, 2017.

20. Ono H, Motoi N, Nagano H, Miyauchi E, UshijimaM, Matsuura M, Okumura S, Nishio M, Hirose T, Inase N and Ishikawa Y: Long noncoding RNA HOTAIR is relevant to cellular proliferation, invasiveness, and clinical relapse in small-cell lung cancer. Cancer Med 3: 632-642, 2014.

21. Cao R, Saracini C, Ginsbach JW, Kieber-Emmons MT, Siegler MA, Solomon EI, Fukuzumi S and Karlin KD: Peroxo and superoxo moieties bound to copper ion: Electron-transfer equilibrium with a small reorganization energy. J Am Chem Soc 138: 7055-7066, 2016.

(i) (3) This work is licensed under a Creative Commons Attribution-NonCommercial-NoDerivatives 4.0 International (CC BY-NC-ND 4.0) License. 